\title{
Design of Sports Training System and Motion Monitoring and Recognition under Wireless Sensor Network
}

\author{
Yue Jia \\ School of Physical Education, Jilin University, Changchun 13000, Jilin, China \\ Correspondence should be addressed to Yue Jia; jiayue@jlu.edu.cn
}

Received 14 August 2021; Revised 16 September 2021; Accepted 28 September 2021; Published 14 October 2021

Academic Editor: Sang-Bing Tsai

Copyright ( 2021 Yue Jia. This is an open access article distributed under the Creative Commons Attribution License, which permits unrestricted use, distribution, and reproduction in any medium, provided the original work is properly cited.

\begin{abstract}
In conventional sports training, coaches record and observe athletes' sports data and judge whether it is reasonable based on their own experience. This qualitative analysis method is highly subjective, has large errors, and is susceptible to interference. To solve the above problems, the design of the sports training system under the wireless sensor network and the research of movement monitoring and recognition become very important. This article aims to study the design of sports training system and the monitoring and recognition of actions under the wireless sensor network technology. This paper simulates the implementation of the proposed data collection protocol and the two basic protocols, the direct transfer algorithm and the flooding algorithm, and compares the protocol proposed in this paper with the other two algorithms in terms of average information transmission success rate and average network overhead. Among them, the average information transmission success rate represents the ratio of the number of messages successfully arriving at the base station to the total amount of information generated by all nodes, and the average network overhead represents the average number of messages sent by each node. Experimental results show that the data collection protocol proposed in this paper can dynamically provide different transmission qualities for information of different importance levels, effectively reducing network overhead, and the reduced overhead is $11 \%$ of the original.
\end{abstract}

\section{Introduction}

1.1. Background. With the progress of society, the pace of work is getting faster and faster. The sedentary work style has caused many people to have physical and mental illnesses, which greatly affects the people's quality of life. Action recognition is to infer the human body's movement state by recording certain movement characteristics of the human body, and then estimate the human body's daily energy consumption, provide suggestions for people's exercise according to the energy consumption, and even guide people to make fitness plans. Through the collaboration between nodes, WSN perceives, collects, and processes the information of the detection object or environment in the monitoring area in real time and delivers the processed information to the end user. WSN has a wide range of applications. It not only has important value in environmental science and military affairs but also has broad application prospects in commercial fields such as daily medical care and smart home.
1.2. Significance. This paper is based on wireless sensor network technology, researches on the design of sports training system and motion monitoring and recognition, proposes an overall design scheme of human motion detection system, and designs the detection terminal of wireless sensor network, which can form a network, collect, and integrate. The data are transmitted, and finally through the host computer, the real-time changes of temperature, heart rate, and exercise energy consumption generated by the human body can be monitored and stored. Through system analysis, it can provide healthy, scientific, and effective suggestions for athletes' sunshine exercise. Introducing information technology into the work of formulating scientific training plans can not only reduce a lot of time spent on coaching statistics and improve work efficiency but also quickly grasp and analyze athletes' training status and complete systematic and detailed experience summaries in time. Input the quantitative parameters of athletes' technical movements into the database and compare with the data of 
the expert database to find out the deficiencies of sports skills and provide the basis for the coaches to formulate training plans.

1.3. Related Work. Movement state recognition not only has important application value in the prevention of chronic diseases but also research on movement state recognition is of great significance for improving human health and improving people's quality of life. Zhao pointed out that the current sports sprint training not only pays attention to speed, strength, and technique but also emphasizes the coordination of lower limb training and upper limb movement. This can meet the needs of current sports sprint training. In response to this problem, the design of the sports sprint training monitoring system can be optimized based on the STC89C51RC microcontroller technology. The results show that the optimization of the design of the sports sprint training monitoring system can help coaches find bad sports habits in the actual sports sprint training process and correct the wrong sprint actions of the athletes in time. The strength data of sports sprint training athletes obtained through the monitoring system can improve the accuracy of the sprint training strength data collection by $30.2 \%$, which shows the benefits of the system application, but the monitoring system has not been fully applied in actual training $[1,2]$. Hong analyzed the energy-saving provided by the collaborative broadcasting form called Large Opportunity Array (OLA) and compared the experiment with the multi-hop performance of the network. He derives an optimal energy distribution strategy, but the components of the strategy are highly expensive [3]. Guo proposed an energysaving distributed scheduling algorithm Clu-DDAS based on a new cluster-based aggregation tree. This method proved the time delay limit, but from the experimental results, the practicability is not very high [4].

1.4. Main Content. This paper focuses on the design of sports training system based on wireless sensor network and the research on movement monitoring and recognition. This article mainly introduces the target tracking method of wireless sensor network and discusses the network architecture of wireless sensor network and its application prospects in sports. The research status of data acquisition technology in wireless sensor networks is analyzed, and the representative data acquisition algorithms in recent years are summarized, and their advantages and disadvantages are analyzed. Behavior monitoring and recognition experiments show that, compared with the existing wireless sensor network data collection protocol, the adaptive data collection protocol has a longer network life and can effectively ensure the success rate and reliability of data transmission. The experimental results show that the data collection protocol proposed in this paper can dynamically provide different transmission qualities for information of different importance levels and effectively reduce network overhead.

\section{Target Tracking Method of Wireless Sensor Network}

2.1. The Coverage of the Monitoring Area by the Network. In order to find the target, a large number of sensor nodes $[5,6]$ are randomly scattered in the area that needs to be detected, and the sensing ranges of the nodes overlap each other, which involves the coverage of the monitoring area. The following first introduces the sensor network coverage model and some concepts involved, and then gives the influence of the detection probability of the target in the monitoring area on the tracking of the moving target.

2.1.1. Basic Concept of Coverage. The coverage problem is determined by the sensing radius of all sensor nodes, communication radius, node energy, and their positional relationship. The union of the sensing conditions of each sensor node constitutes a description of the coverage of the entire sensing area. The wireless sensor network coverage model is shown in Figure 1.

In the figure, the solid point represents the sensor node, Rs represents the sensing radius, the sensing radius refers to the farthest distance of the target that a single sensor node can perceive, sometimes called the detection radius, the larger the Rs, the larger the area of the monitoring area. The outer boundary composed of the union of circles is the monitoring area covered by the wireless sensor network [7].

The size of its coverage area is covered by a single node. The coverage must be less than or equal to 1 . If all locations in area $A$ are covered, the coverage is 1 .

$$
\mathrm{CP}=\frac{\cup_{i=1 \ldots N} A_{i}}{A} .
$$

The calculation of coverage efficiency CE is shown in formula (2):

$$
\mathrm{CE}=\frac{\underset{i=1 \ldots N}{\cup} A_{i}}{\sum_{i=1 \ldots N} A_{i}} .
$$

According to the definition of coverage efficiency, it is not difficult to find that efficiency also reflects the degree of redundancy of nodes. The higher the coverage efficiency, the lower the redundancy, and the opposite, the greater the node redundancy.

Coverage efficiency is used to measure the utilization rate of node coverage. On the one hand, it can reflect the coverage situation, and on the other hand, it can reflect the energy consumption of the entire network $[8,9]$.

Coverage dimension: coverage dimension represents the degree of redundancy of coverage of a certain area.

$$
K_{A}=\sum_{i=1}^{N} K_{i}, K_{i}= \begin{cases}1, & P_{a} \subseteq A_{i}, \\ 0, & P_{a} \cap A_{i} \neq P_{\text {node }} .\end{cases}
$$

In the formula, $K_{A}$ : the coverage dimension of area, $A, A_{i}$ : the perception range of the node, and $P_{a}$ : the position of the analysis point. 


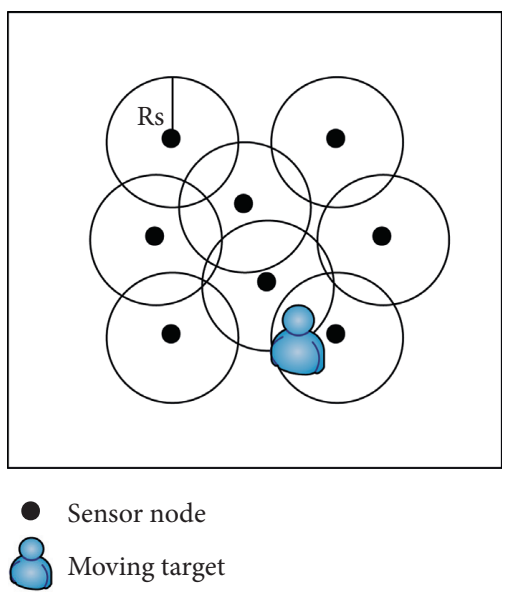

FIGURE 1: Schematic diagram of network coverage model.

In order to provide strong monitoring capabilities and higher fault tolerance for the target tracking monitoring area, the monitoring area is often covered by multiple coverage, that is, a target is covered by $k$ nodes.

2.1.2. Distribution of Covered Nodes. Coverage is directly related to the distribution of nodes, the number of nodes, and the amount of redundancy in the monitoring area. The distribution of nodes will directly affect the coverage efficiency.

(1) Deterministic Distribution. In deterministic distribution of nodes for the network coverage problem, many problems are abstracted as static optimization problems, which brings some convenience to solve the problem, but in practical applications, especially in the harsh environment of largescale, unsupervised, it cannot be determined.

(2) Random Distribution [10, 11]. The nodes in theoretical research are generally randomly distributed, and different random distribution forms can be adopted according to the addition of application requirements, the upper uniform distribution, and the restriction of node density. Compared with random distribution, the reason for not adopting deterministic distribution lies in practical reasons. Deterministic distribution cannot be achieved in a large-scale, unsupervised environment.

(3) Removable Distribution. Mobile distribution is divided into two types: all nodes can be moved and part of them can be moved. The former has a higher degree of freedom, which will increase energy consumption while bringing high coverage quality. The latter model is a hybrid model, which makes up for the static node coverage problem, while also bringing flexibility to the network.

2.1.3. Analysis of Target Detection Probability. When sensor nodes are deployed in the monitoring area, they will spontaneously monitor target information. Due to the uncertainty of sensor node distribution, there is no guarantee that the target will be $100 \%$ discovered. The target detection probability is an estimate of the detection probability of the monitoring area. According to the above analysis, if the target is within the sensing radius covered by the node $[12,13]$, then the target will be detected. On the contrary, the target will not be detected if it is outside the sensing radius covered by the node.

Taking the node distribution in Figure 1 as the research object, the monitoring area in the figure is $\mathrm{A}$, and the sensing area of each node [11] is $\prod R_{s}^{2}$. Then the detection probability $P$ of the node to the target in the rectangular area can be expressed as

$$
P=\frac{\prod R_{S}^{2}}{A .}
$$

The probability that the node does not detect the target is $1-P$.

Assuming that the number of nodes in $A$ obeys the Poisson distribution, the probability of $k$ nodes in area $A$ is

$$
P(k)=\frac{e^{-\lambda} \times \lambda^{k}}{k !}, \quad k=1,2, \ldots+\infty .
$$

There are $k$ nodes in the sensing area, and the probability of at least one node detecting the target is

$$
\mathrm{DP}(k)=\frac{e^{-\lambda} \times \lambda^{k}}{k !}\left(1-(1-P)^{k}\right) .
$$

According to the total probability formula, when $K$ takes different values, the probability DP of at least one node detecting the target can be expressed as

$$
\operatorname{DP}(k)=\sum_{k=1}^{\infty} \frac{e^{-\lambda} \times \lambda^{k}}{k !}\left(1-(1-p)^{k}\right) .
$$

Because

$$
\begin{aligned}
\sum_{k=1}^{\infty} \frac{e^{-\lambda} \times \lambda^{k}}{k !}=\sum_{k=0}^{\infty} \frac{e^{-\lambda} \times \lambda^{k}}{k !}-\frac{e^{-\lambda} \times \lambda^{0}}{0 !} & =1-e^{-\lambda} \\
\sum_{k=1}^{\infty} \frac{e^{-\lambda} \times \lambda^{k}(1-P)^{k}}{k !} & =e^{-\lambda P}-e^{-\lambda} .
\end{aligned}
$$

Substituting formula (8) and (9) into formula (7), we can get

$$
\mathrm{DP}=\left(1-e^{-\lambda}\right)\left(e^{-\lambda P}-e^{-\lambda}\right)=1-e^{-\lambda P} .
$$

Substituting (4) into formula (10), the expression of target detection probability [14] is finally obtained as follows:

$$
\mathrm{DP}=1-e^{-\lambda P}=1-e^{-d \times A \times\left(\prod R_{s}^{2} / A\right)=1-e^{-d \prod R_{s}^{2} .}}
$$

From formula (11), it can be seen that the target detection probability has an exponential relationship with the node density and the node's perception radius. The detection probability will increase as the nodes are dense and the sensing radius increases. At the same time, the analysis of detection probability also has a guiding role in node placement. As the nodes are dense and the sensing radius increases, the detection accuracy and range are improved. 
The sensing radius Rs is determined by the accuracy of the sensor node and the amplification factor of the amplifier, which can be regarded as a known quantity here.

2.2. Target Tracking Node Selection. The target tracking node [15] is dynamically selected with the moving target. After initialization, the monitoring area nodes are all ordinary nodes. When the moving target is in the tracking area, the ordinary node close to the moving target becomes the target tracking node, and it needs to track the moving target for a period of time, and then return to the normal node state. This section mainly discusses how to choose the selection process.

2.2.1. Detection of Target Tracking Nodes. When selecting target tracking nodes, cluster head nodes and common nodes are required to cooperate. Similarly, the cluster head node should also consider the following two situations: first, the target tracking node should always track the movement of the target and wake up the nodes near the prediction area and high energy consumption. The cluster head node should periodically detect the energy of the tracking node and replace the tracking node to avoid energy consumption of the tracking node and target tracking failure. Second, when the target tracking node suddenly fails during the working process, such as the node is damaged, the cluster head node should be able to deal with it in time.

First, set an energy threshold and the number of times that the node is a target tracking node. The node is in a periodic sleep and wake-up mechanism. The conditions for the node to become a tracking node must be met: greater than the energy threshold and the node position is within the distance of the moving target-hop.

In order to cooperate with different positioning methods, distance and angle factors should also be taken into consideration. The tracking node selection model [16] is given as follows:

$$
f(n, e, d, t)=-A_{1} \times n+A_{2} \times e+A_{3} \times d+A_{4} \times t .
$$

In the formula: $A_{1}, A_{2}, A_{3}$, and $A_{4}$ are, respectively, the target tracking node, the remaining energy of the node, the distance to the moving target, and the coefficient of the angle between the moving target, which are determined by the specific application.

The specific selection process is as follows: before the start of the node work cycle, all nodes within one hop of the moving target check their own remaining energy, and the nodes higher than the threshold calculate the degree of selection through (12) and apply to the cluster head node to become a tracking node. After receiving the application of the node, the cluster head node compares the degree of selection of each node and sends a message of "determine tracking node" to the selected node, and the node that is not selected sends a message of "cancel tracking node" and repeat for the next work cycle.

In the working process, if the tracking node suddenly dies, the cluster head node needs to periodically check the data sent by the node during the working process of the tracking node. If the tracking node does not send data or sends wrong data several times in a row, the cluster head node needs to replace the working node. Broadcast the replacement message to notify other nodes. Other nodes again calculate their own selection degree to compete for tracking nodes.

2.2.2. Selection Process of Target Tracking Node. Based on the above analysis, the complete process of target tracking node selection [17] is shown in Figure 2.

2.2.3. Dynamic Time Bending. DTW is an effective method to measure the distance between two time series data. Suppose that there are two time series data $Q$ and $C$ with lengths $m$ and $n$ :

$$
\begin{aligned}
& Q=\left(q_{1}, q_{2}, \ldots, q_{m}\right), \\
& C=\left(c_{1}, c_{2}, \ldots, c_{n}\right) .
\end{aligned}
$$

When the lengths of $Q$ and $C$ are the same, the most primitive way to measure distance is

$$
D=\sum_{i=1}^{n}\left|\left(q_{i}-c_{i}\right)\right| .
$$

Solve the distance between each two sampled data points in a one-to-one correspondence and add the distance between each pair of points to get the distance between the two time series data. This calculation method is very simple and clear, and the calculation is complicated. However, when the two time series data are shifted or distorted, this one-to-one correspondence calculation method will make the distance between the two originally very similar curves very large. And when the lengths of the two data sequences are not equal, this one-to-one correspondence calculation method cannot be used.

The DTW algorithm can effectively solve the above time series data translation and distortion problems, that is, even if two similar curves have a certain translation and distortion [18], the DTW distance is still very small, and the DTW algorithm is also suitable for two time series. The length of the data is inconsistent.

\subsection{Construction and Update of Target Tracking Area.} After completing the target estimation prediction and tracking node selection, it is necessary to construct a tracking area near the predicted trajectory. Because the target is mobile, the tracking area needs to be continuously updated to achieve the tracking of the moving target. The update of the tracking area is usually related to the speed of the moving target. If the target tracking area can be updated reasonably according to the characteristics of the moving target, the limited resources of the node can be fully deployed and utilized, and the system performance can be effectively improved.

Since the target is in motion, it may move to a position outside the tracking area after a period of time. The tracking area needs to be continuously updated according to the 


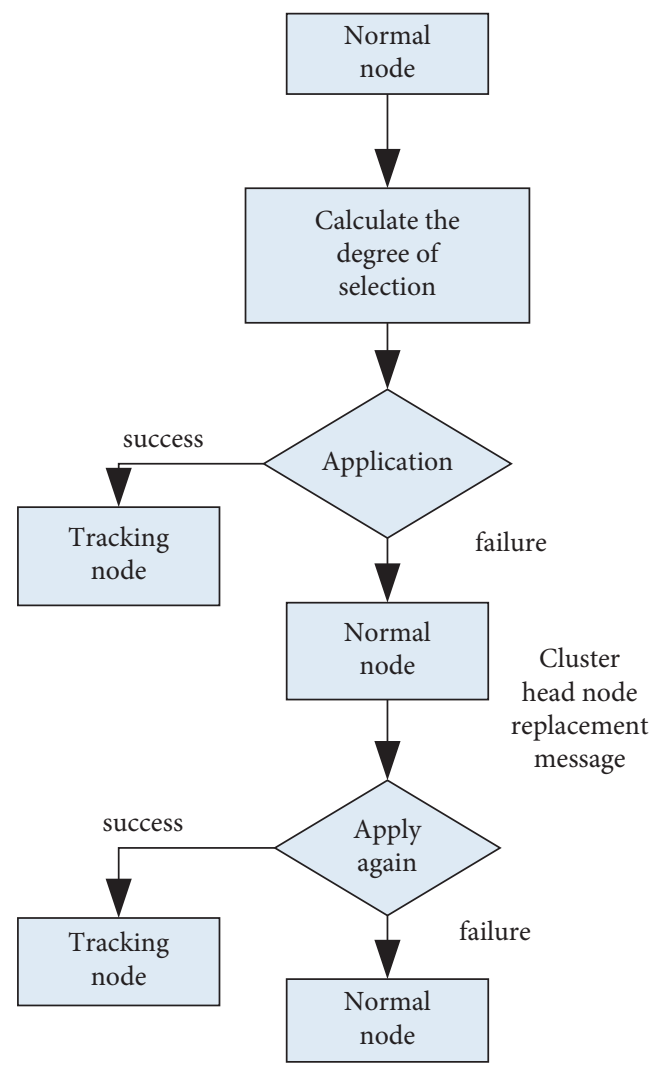

(a)

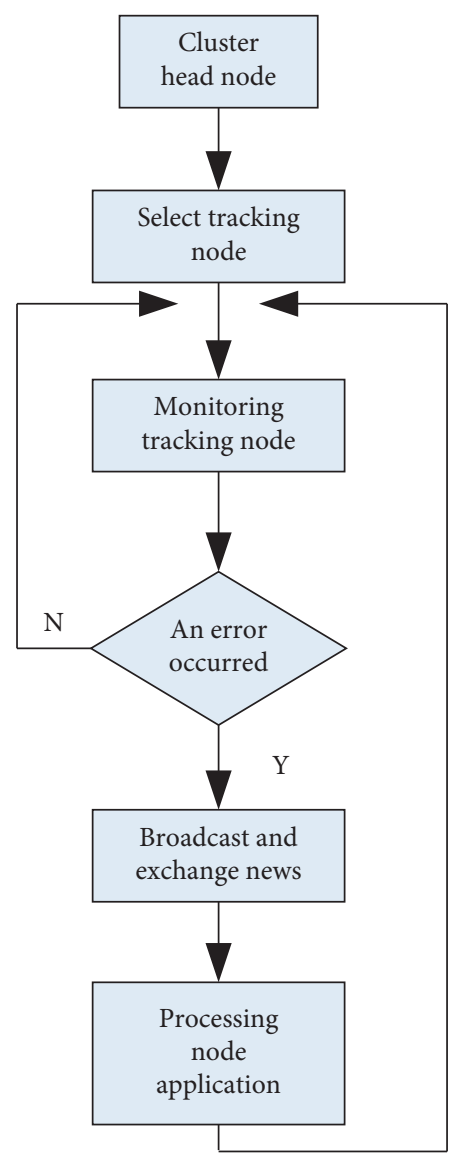

(b)

FIgURE 2: Flowchart of target tracking node selection. (a) Ordinary node workflow. (b) Common node workflow.

target's motion status. Assuming that $T_{\text {fre }}[19]$ is the life cycle of the tracking area, if it exceeds the $T_{\text {fre }}$ tracking area and is not updated, it may cause the tracking of the moving target to be lost.

$$
T_{\text {fre }}=\frac{S}{v_{\max }}=2 \frac{\prod L}{v_{\max } \tan \varphi_{\max }}
$$

Before the life cycle of the current tracking area ends, the next tracking area needs to be constructed, and then the corresponding node is notified to complete the construction. According to the analysis, this process can be divided into three stages, calculate the life cycle of the area (T1), determine the next tracking area range (T2), and notify the corresponding node (T3). The first stage takes a relatively short time, and it can also be estimated from the previous tracking area, and then this area is notified. The second and third stages need to add specific considerations, such as moving target speed, node data transmission speed, network topology, and other factors.

According to the above formula to complete the target tracking area estimation, the cluster head node [20] will determine which nodes are in the tracking area, and then send a message to notify these nodes to enter the tracking phase, and the message contains the predicted location of the target and the range of the tracking area.

\section{Design Experiment of Sports Training System}

3.1. Performance Evaluation Index of Data Collection Algorithm. Regarding the performance of wireless sensor network data collection protocols [21], there are the following commonly used evaluation indicators:

3.1.1. Data Accuracy. The data accuracy requirements of the data collection algorithm depend on the application background of the sensor network and user needs. For example, indoor temperature and humidity monitoring systems do not require high data accuracy. However, battlefield target tracking in military applications requires high data accuracy to ensure accurate strikes $[22,23]$.

3.1.2. Scalability. A typical wireless sensor network contains thousands or even more sensor nodes, the network scale is large, and the scalability problem of the data collection protocol is very prominent.

3.1.3. Response Time. The characteristic of scalability is the response time. Response time includes data transmission, routing, and data fusion time. It is represented by the time delay between sending sensor data from the target node and 
receiving data messages from the base station. Different applications have different requirements for real-time data, and a reasonable data collection algorithm [24] is designed according to the needs.

Robustness, that is, the robustness of the network, the characteristic that the network can maintain its own performance when its own and environmental parameters change.

The above-mentioned performance index is not only the standard for evaluating the data collection algorithm of wireless sensor network but also the goal of algorithm design optimization. However, the above indicators are not completely compatible in practical applications and must be balanced with each other to achieve algorithm optimization.

The topology of the wireless sensor network is an important factor that affects the performance of the data collection protocol. There is a close relationship between data transmission capacity and energy consumption in different network structures. The plane routing is simple and stable, but the routing establishment and maintenance are expensive, and it is suitable for small- and medium-sized networks. Hierarchical routing has good scalability, can facilitate data fusion, and is suitable for large-scale networks. Several representative flat and hierarchical data collection protocols are introduced as shown in Table 1:

\subsection{Data Collection Mechanism in the Exercise System.} The design of data collection in wireless sensor networks is closely related to specific applications, and the particularity of the application environment is an issue that needs to be considered. In this article, for the specific application of sports training, the following points need to be paid attention to when designing the data collection protocol.

This article adopts the method based on wireless sensor network to monitor the athlete's sports parameters. Most monitored objects are in motion, which requires sensor nodes to be movable. Since the movement of the monitored object will cause the dynamic change of the sensor network topology and the discontinuity of the network connection, the above influence must be fully considered when designing the data collection protocol to ensure that the network has high scalability.

Athlete parameter monitoring is a long-term work, and sensor nodes need to be able to work effectively for a long time. The energy consumption of nodes should be fully considered when designing the data collection protocol. The data collection algorithm should try to meet the energy-saving requirements. Without affecting the performance of the entire network, the method of combining with other energy-saving strategies is adopted to reduce energy consumption [25].

\subsection{Overall Framework of Motion System Based on Wireless} Sensor Network. Figure 3 is a schematic diagram of the overall structure of the sports system. The sports system monitors multiple sports and physiological parameters of the athletes through wireless communication. The sports data collected by the base station can be stored in the database system for a long time to provide a basis for the comprehensive sports analysis of outstanding athletes and construct an expert system. The mobile system mainly includes three parts: node, base station, and data management center.

3.3.1. Node. The main function of the wireless sensor network node is to collect data in real time and send the data to the base station after storage. In this system, nodes mainly collect athletes' motion parameters and physiological parameters, and then wait for the query instruction from the base station or perform periodic data reporting. When the node receives the query instruction from the base station, the node sends the collected data to the base station. In actual training, the node turns on its own LED or buzzer according to the instructions of the base station to assist athletes in completing training tasks while collecting data.

3.3.2. Base Station. The main role of the base station is to be responsible for system networking, receive and process data sent by the node, and send instructions to the node according to the user's requirements. The data received by the base station are mainly processed in the following two ways. The base station sends the data to the data management center server through the serial communication module or the Ethernet communication module. Coaches and employees can browse and manage the data through the data center management software. Direct display of data is through the base station LCD touch screen. In order to facilitate the coaches to inquire and control the athlete's skill information and sports test training at the sports training site, the coaches on the training field hold the base station, control the working status of the node by touching the LCD screen, and browse the sports test information of the athletes.

3.3.3. Data Management Center. Since the base station can transmit data through the network, it can automatically transmit the data to the Internet. Therefore, the data management center in this system can be deployed in any geographical location, as long as the network is connected. Coaches can also view the related changes of athletes' sports parameters through the sports database management system software and monitor the operation of the wireless sensor network in real time. For example, athletes wear sports watches, and coaches use system software to view specific sports information.

3.4. Motion Monitoring and Recognition Experiment. In order to verify the effectiveness of the double-layer cooperation mechanism, the program uses $\mathrm{VC}++$ to simulate the movement trajectory of the moving target in different monitoring environments.

3.4.1. Adaptability Analysis. In the area where the monitoring area is $1000 * 1000$, the communication radius is 150 meters, the number of delivery nodes is from 500 to 50,10 
TABLE 1: Comparison of data collection algorithms based on network structure.

\begin{tabular}{|c|c|c|c|c|c|c|}
\hline Protocol & $\begin{array}{l}\text { Network } \\
\text { structure }\end{array}$ & Scope of application & $\begin{array}{l}\text { Data } \\
\text { fusion }\end{array}$ & $\begin{array}{l}\text { Energy- } \\
\text { saving }\end{array}$ & Delay & $\begin{array}{c}\text { Number of } \\
\text { paths }\end{array}$ \\
\hline $\mathrm{DD}$ & Flat type & $\begin{array}{l}\text { Small-scale network driven by periodic queries with } \\
\text { few aggregation nodes }\end{array}$ & $\mathrm{V}$ & Difference & High & $\begin{array}{c}\text { Multi- } \\
\text { diameter }\end{array}$ \\
\hline SPIN & Flat type & $\begin{array}{l}\text { More sink nodes, source nodes } \\
\text { Fewer small-scale networks }\end{array}$ & $\mathrm{V}$ & Good & General & $\begin{array}{l}\text { Multi- } \\
\text { diameter }\end{array}$ \\
\hline LEACH & Hierarchical & $\begin{array}{l}\text { Small- and medium-sized networks with high node } \\
\text { energy }\end{array}$ & $\mathrm{V}$ & Difference & General & Single path \\
\hline TEED & Hierarchical & $\begin{array}{c}\text { Small- and medium-sized with higher node energy } \\
\text { The Internet }\end{array}$ & $\mathrm{V}$ & Well & Low & Single path \\
\hline CLUDDA & Hierarchical & Network with higher node energy & $\mathrm{V}$ & Good & Low & Single path \\
\hline PEGASIS & Chain type & $\begin{array}{l}\text { Small- and medium-sized networks with high node } \\
\text { energy }\end{array}$ & $\mathrm{V}$ & Good & High & Single path \\
\hline EADAT & Tree type & $\begin{array}{l}\text { Single sink node, periodic } \\
\text { query-driven small-scale networks }\end{array}$ & $\mathrm{V}$ & Good & Low & Single path \\
\hline
\end{tabular}

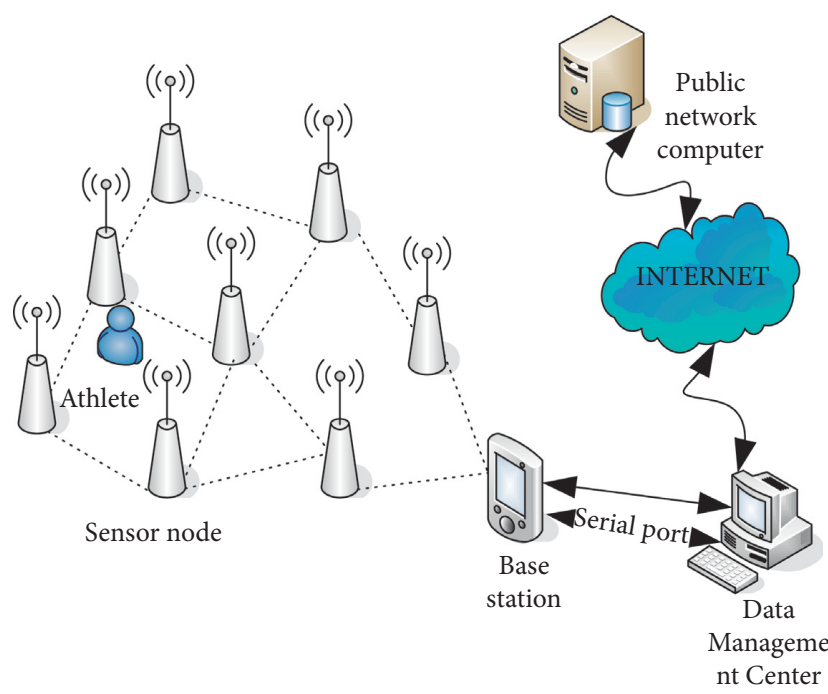

FIgURE 3: Schematic diagram of the system structure.

locations are randomly tracked, and less than 3 nodes are defined as unable to be located. Under the same conditions, compare the influence of the scheme and DCTC on positioning when the density changes.

It can be seen from Table 2 that as the number of nodes increases, the positioning accuracy of the two schemes is basically the same, but this scheme has obvious advantages in terms of calculation time and energy consumption. This solution can complete tracking and positioning when the node density exceeds $1 \%$.

3.4.2. Energy Consumption Analysis. Assuming that the speed and trajectory of the moving target are the same, this scheme is compared with DCTC, and the energy consumption is analyzed in two aspects. First, the node participation in target tracking: count the number of nodes awakened in the tracking area, working time, and sending and receiving time to determine the difference in energy consumption between the two schemes.
(1) Experimental Conditions: The node density is $2 \%$, the moving speed is $10 \mathrm{M} / \mathrm{s}$, and the average energy consumption is tested 10 times. Assumptions: the energy consumption of the sleeping node is $0.001 \mu \mathrm{J}$, the energy consumption of the working node is $0.3 \mathrm{~J}$, and the energy consumption of the transceiver node is $1.12 \mathrm{~J}$.

Through the analysis of the operating conditions of the entire network nodes, the experimental results are shown in Table 3 . The operating energy consumption of this scheme is only $28.9 \%$ of the DCTC energy consumption. The second is the average reconstruction time. The comparison result of these two schemes is shown in Figure 4.

The calculation of average reconstruction time: the DCTC algorithm requires $8.5 \mathrm{~s}$, the average reconstruction time of this scheme is $12.4 \mathrm{~s}$, because the speed normalization calculation method can be obtained, and this scheme can be obtained according to the speed normalization method, which is better than DCTC. The plan was reduced by $31.3 \%$.

3.4.3. Analysis of Trajectory Positioning Accuracy. In the experiment, the DCTC, this scheme, and the random positioning method were used to compare the orbit positioning accuracy. The experimental results are shown in Figure 5. Compared with the DCTC positioning method, the positioning accuracy of this method is significantly better than that of the DCTC scheme.

\section{Performance Analysis}

4.1. Performance Comparison Analysis. This paper simulates and implements the proposed data collection protocol and the two basic protocols Direct Transmission and Flooding. The performance of the three protocols is compared through the following two aspects:

(1) Compare the average information transmission success rate and average network overhead between the protocol proposed in this paper and the other two algorithms. In order to verify that information of different importance levels provides different 
TABLE 2: The impact of node density changes on positioning.

\begin{tabular}{lcccccc}
\hline $\begin{array}{l}\text { Program total } \\
\text { number of nodes }\end{array}$ & $\begin{array}{c}\text { 50-Node } \\
\text { positioning } \\
\text { accuracy }\end{array}$ & $\begin{array}{c}\text { 70-Node } \\
\text { positioning } \\
\text { accuracy }\end{array}$ & $\begin{array}{c}\text { 90-Node } \\
\text { positioning } \\
\text { accuracy }\end{array}$ & $\begin{array}{c}\text { 100-Node } \\
\text { positioning } \\
\text { accuracy }\end{array}$ & $\begin{array}{c}\text { 500-Node } \\
\text { positioning } \\
\text { accuracy }\end{array}$ & $\begin{array}{c}800 \text {-Node } \\
\text { positioning } \\
\text { accuracy }\end{array}$ \\
\hline This plan & 11 & 10 & 8 & 6 & 5 & 5 \\
DCTC & 10 & 7 & 5 & 5 & 4 & 4 \\
\hline
\end{tabular}

transmission quality, the experiment separately studied the changes in the average transmission success rate of information when the initial importance levels were $0.4,0.6$, and 0.8 . Since different importance levels have very little impact on the average network overhead, the impact of the importance level is ignored when comparing the average network overhead. The average information transmission success rate refers to adding up all the information transmission success rates and then taking the average, while the average network overhead is the average value after adding the network overhead.

(2) The effects on the performance of the three algorithms under three different experimental parameters: node moving speed, node queue space size, and the number of base stations in the network are separately studied. In the direct delivery algorithm, each node buffers all the information collected by itself until it encounters the base station and forwards it. In the flooding algorithm, each time two nodes communicate, they forward information that is not in the queue of the other party. When a node encounters a base station, it forwards all the information. In order to evaluate the pros and cons of the proposed adaptive data collection protocol, we wrote the simulation process in $\mathrm{C}++$, and the simulation environment was set as follows:

We select a $500 \mathrm{~m} 500 \mathrm{~m} \times$ square area as the deployment range of the mobile sensor network, including 150 nodes and 5 base stations, where the nodes move according to the random motion model, and the base stations remain stationary. The whole area is divided into independent squares of $50 \mathrm{~m} 50 \mathrm{~m} \times$, the moving speed of the node obeys a random distribution of $1 \mathrm{~m} / \mathrm{s} 10 \mathrm{~m} / \mathrm{s}-$, and the pause time of each node after reaching a new position obeys a random distribution of $0 \mathrm{~s} 10 \mathrm{~s}-$. The node generates a new message every 20 seconds, the size of the message is 20Byte, and the initial importance of the new message is randomly selected according to $[0.4,0.6,0.8]$. The queue of the node can hold up to 300 messages, the communication radius of the node is $20 \mathrm{~m}$, and the bandwidth of the channel is $25 \mathrm{kbit} / \mathrm{s}$.

\subsection{The Impact of Node Moving Speed on Performance}

4.2.1. The Effect of Node Moving Speed on the Average Success Rate of Information Transmission. The effect of node movement speed on the average information transmission success rate is shown in Figure 6. As the node movement speed increases, the average information transmission success rate of the protocol and direct delivery algorithm in this article continues to increase, while the average information transmission success rate of the flooding algorithm gradually increases. In this protocol and the direct sending algorithm, as the moving speed of nodes increases, communication opportunities between nodes and between nodes and base stations increase, which requires forwarding nodes to have more opportunities to select appropriate relay nodes to forward information. In addition, it can be seen from the figure that the initial importance still affects the average success rate of information transmission.

4.2.2. The Impact of Node Movement Speed on Average Network Overhead. The influence of node moving speed on average network overhead is shown in Figure 7. As the node moving speed increases, the average network overhead of this protocol increases slightly. Since the communication radius of the node is fixed within $20 \mathrm{~m}$, with the increase of the node's moving speed, the network topology change is accelerating, and the node's encounter probability and transmission probability are also constantly changing. Part of the information will be forwarded back and forth between different nodes, thus increasing the node information transmission frequency.

\subsection{The Impact of Node Queue Length on Performance}

4.3.1. The Effect of Node Queue Length on the Average Success Rate of Information Transmission. The effect of node queue length on the average information transmission success rate is shown in Figure 8. From the results in the figure, it can be seen that as the node queue length increases, the average information transmission success rate of the direct delivery algorithm does not change much. With the increase of the queue length of the node, the performance of the flooding algorithm is relatively improved. Due to the increase of the queue space, the node can store more information, and it also increases the probability of a single message reaching the base station. The average information transmission success rate of the protocol in this paper increases with the increase of the queue length, and the improvement range is more than that of the previous two algorithms. The main reason is that the protocol in this paper has an efficient queue management strategy. In addition, when the length of the node queue is short, it is easy to overflow and information discarding. Only when the length of the node queue increases to a certain size, the initial importance will affect the average transmission success rate of information, and there will be a big gap. 
TABLE 3: Node participation in target tracking.

\begin{tabular}{lcccc}
\hline $\begin{array}{l}\text { Program total number } \\
\text { of nodes }\end{array}$ & $\begin{array}{c}\text { Total number of } \\
\text { sleeping nodes }\end{array}$ & $\begin{array}{c}\text { Total number of } \\
\text { working nodes }\end{array}$ & $\begin{array}{c}\text { Total number of sending and } \\
\text { receiving nodes }\end{array}$ & $\begin{array}{c}\text { Average energy } \\
\text { consumption } \mu \mathrm{J}\end{array}$ \\
\hline This plan & 192 & 8 & 8 & 11.55 \\
DCTC & 172 & 28 & 28 & 39.93 \\
\hline
\end{tabular}

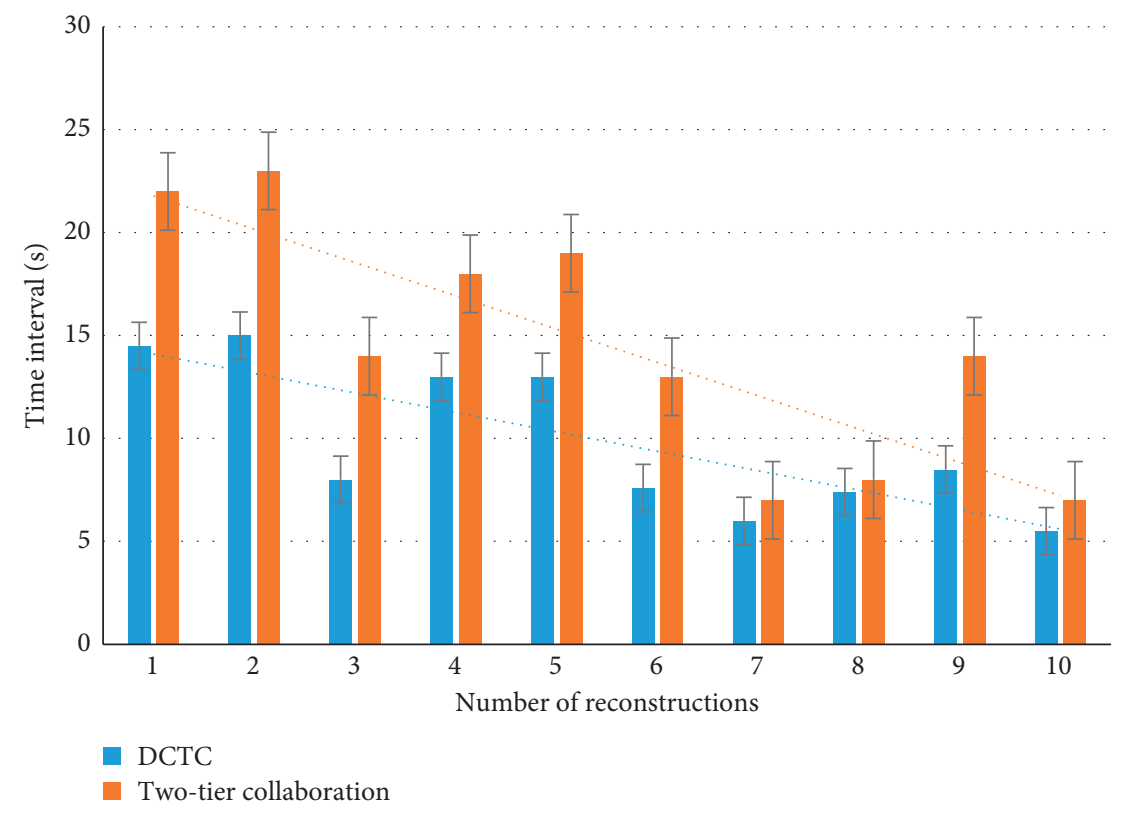

FIgURE 4: Reconstruction of tracking cluster time interval.



FIGURE 5: Comparison chart of trajectory positioning accuracy.

4.3.2. The Effect of Node Queue Length on Average Network Overhead. The effect of node queue length on the average network overhead is shown in Figure 9. The communication overhead of this protocol increases slightly with the growth of the node queue, indicating that the efficient queue management strategy of this protocol can well control the network overhead and the flooding algorithm. The average network overhead rises sharply as the queue grows. This is because the node has more space to store more information and is sent to the network. 




FIgURE 6: The effect of node moving speed on the average transmission success rate.

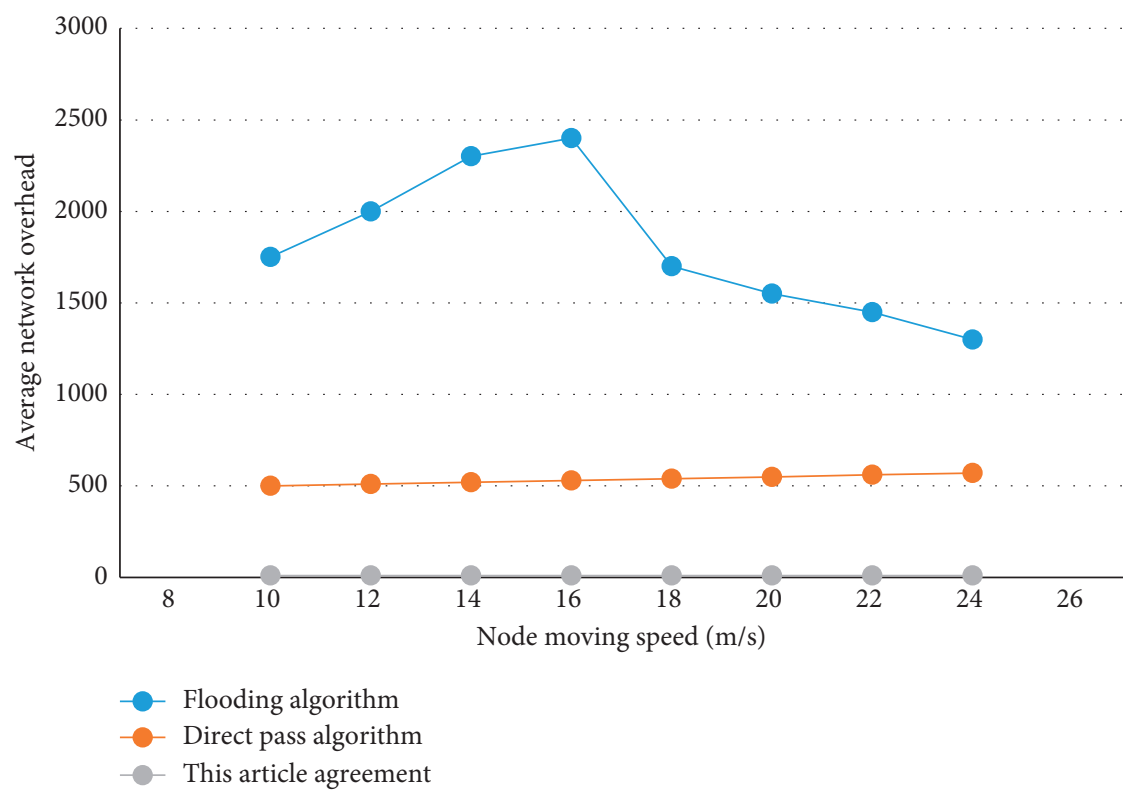

Figure 7: The effect of node moving speed on average network overhead.

\subsection{The Impact of the Number of Base Stations on Performance}

4.4.1. The Influence of the Number of Base Stations on the Average Success Rate of Information Transmission. Figure 10 shows the effect of the number of base stations on the average information transmission success rate. When the number of base stations changes, the protocol proposed in this paper has a better average information transmission success rate than the other two algorithms. When the initial importance degree has a greater impact on the average information transmission success rate, the greater the initial importance degree value, the higher the average information transmission success rate. When the number of base stations is large, the probability of nodes encountering base stations is higher. Therefore, the performance of the protocol proposed in this paper is similar to that of the direct delivery algorithm, and the average information transmission success rate can reach the highest $100 \%$. However, the average information transmission success rate of the flooding algorithm increases with the increase in the number of base stations, but it is much lower than the previous two protocols. The reason is that the information produces many copies of information during the flooding process, and the node's queue space is limited, leading to information overflow and packet discarding.

4.4.2. The Influence of the Number of Base Stations on the Average Network Overhead. Figure 11 shows the effect of the number of base stations on the average network overhead of the three algorithms. It can be seen from the figure that the average number of messages sent by each node of the direct 


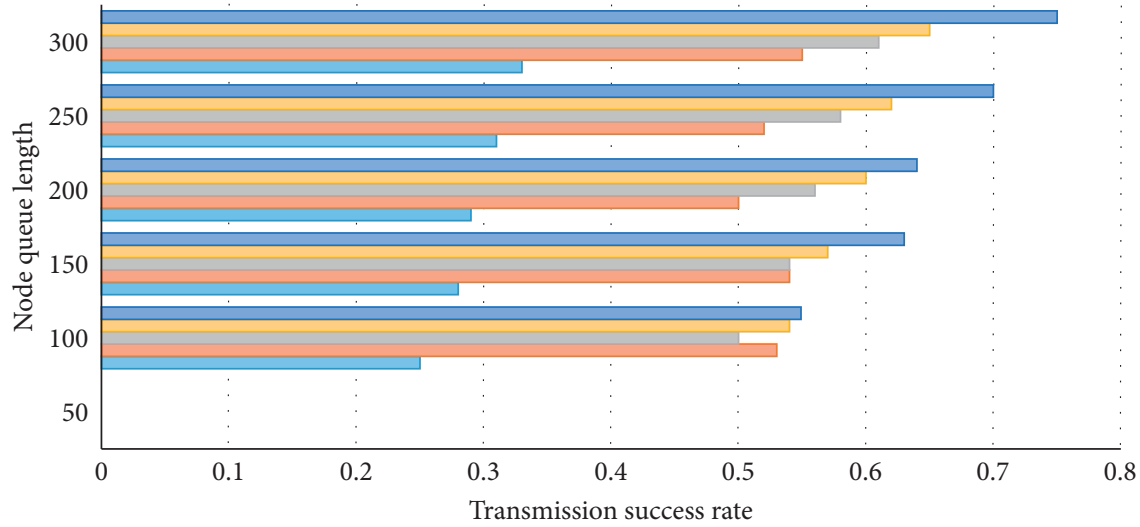

$\square$ This article agreement_0.8

$\square$ This article agreement_0.6

$\square$ This article agreement_0.4

$\square$ Direct pass algorithm

$\square$ Flooding algorithm

FIgURE 8: The effect of node queue length on transmission success rate.



Figure 9: The effect of node queue length on network overhead.

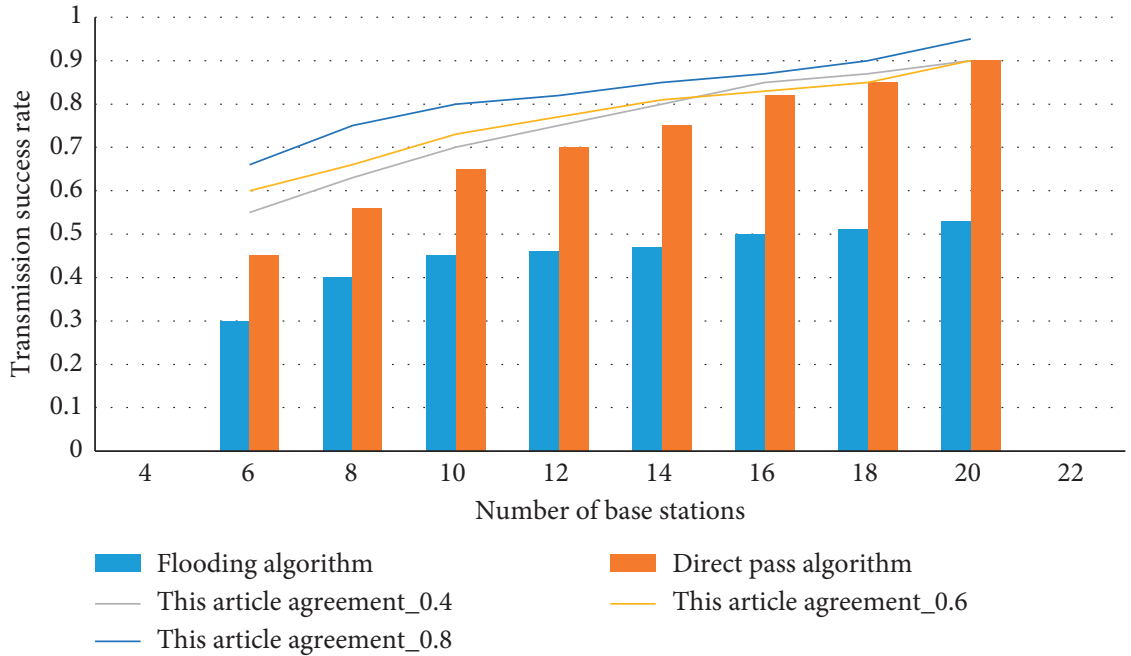

Figure 10: The influence of the number of base stations on the transmission success rate. 


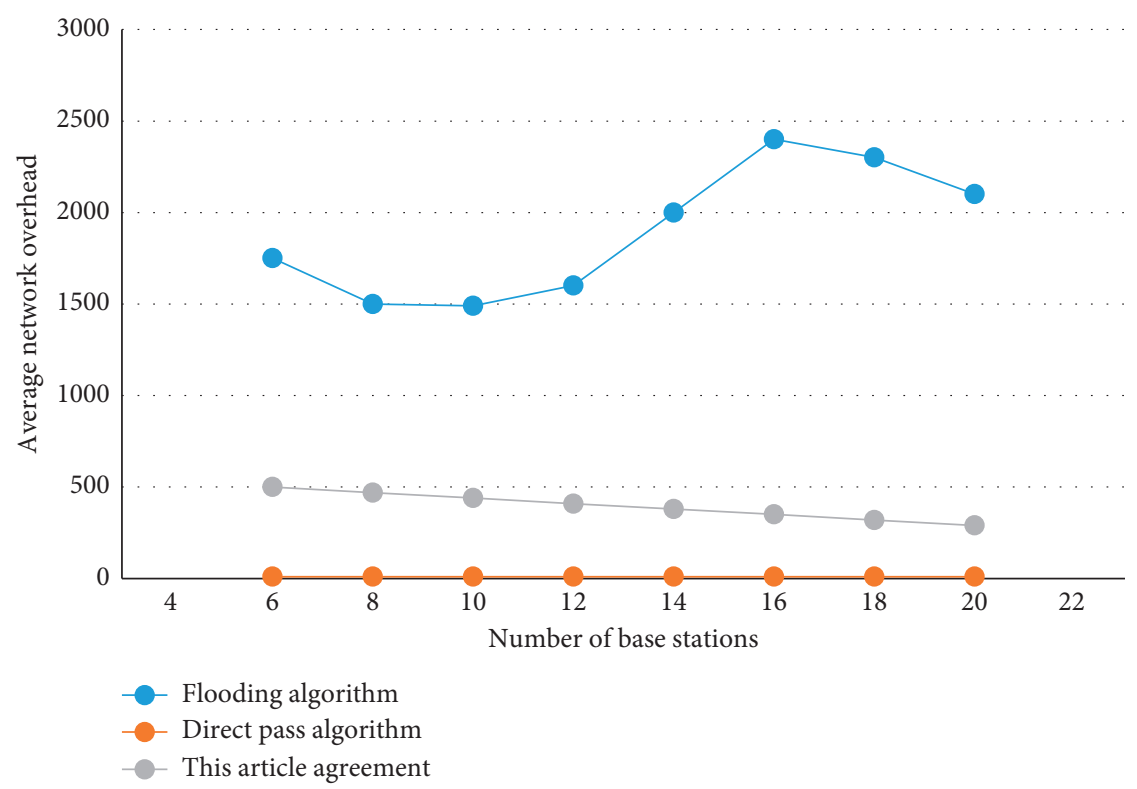

Figure 11: The impact of the number of base stations on network overhead.

delivery algorithm is the least, and the average network overhead is also lower. This is because each node in the direct delivery algorithm basically only sends information generated by itself. In the flooding algorithm, the average number of messages sent by a node is much higher than that of the other two algorithms. As for the protocol proposed in this article, as the number of base stations increases, the average network overhead of nodes decreases slightly. There are two reasons: first, the more base stations deployed, the greater the communication opportunities between the nodes and the base stations, and thus higher transmission probability. Second, the number of hops for information from the source node to the base station decreases, and the average amount of information forwarded by the node decreases.

This chapter proposes an adaptive data collection protocol for mobile sensor networks, including two parts: information transmission and queue management. Compared with the existing wireless sensor network data collection protocol, it can meet different forwarding service quality requirements and is suitable for mobile data collection occasions with multiple types of parameters. The simulation experiment results show that the data collection protocol proposed in this paper can dynamically provide information of different importance levels. Different transmission quality effectively reduces network overhead.

\section{Conclusions}

The research results of wireless sensor networks have obvious application prospects and potential industrial economic value in epidemic disease prevention, disaster warning, national defense, and environmental protection. The research results have obvious application prospects in disease prevention, disaster warning, and other aspects and have potential industrial economic value. Based on the latest development of the current wireless sensor network, based on the practical application of sports, with the support of the special research fund of the Ministry of Science and Technology, this paper conducts research on the design of the sports training system based on the wireless sensor network and the movement monitoring and recognition. It focuses on the network structure of wireless sensor network and its application prospects in the field of sports. The research status of wireless sensor network data acquisition technology is analyzed, and the representative data acquisition algorithms in recent years are summarized. Based on the summary and analysis of the existing wireless sensor network data collection mechanism, a mobile sensor network adaptive data collection mechanism suitable for moving target data collection is proposed. The basic idea is to dynamically copy information to sensor nodes that may communicate with the base station, so as to maximize the transmission success rate and reduce network overhead.

\section{Data Availability}

No data were used to support this study.

\section{Conflicts of Interest}

The author declares that there are no conflicts of interest.

\section{References}

[1] Y. Zhao, "The design and implementation of sports sprint training monitoring system," Revista de la Facultad de Ingenieria, vol. 32, no. 4, pp. 597-604, 2017.

[2] S. Li, C. Chunwei, Zhang, and Tianqi, "The strain transfer mechanism of fiber bragg grating sensor for extra large strain monitoring," Sensors, vol. 19, no. 8, p. 1851, 2019.

[3] Y. W. Hong and A. Scaglione, "Energy-efficient broadcasting with cooperative transmissions in wireless sensor networks," 
IEEE Transactions on Wireless Communications, vol. 5, no. 10, pp. 2844-2855, 2016.

[4] L. Guo, Y. Li, and Z. Cai, "Minimum-latency aggregation scheduling in wireless sensor network," Journal of Combinatorial Optimization, vol. 31, no. 1, pp. 279-310, 2016.

[5] A. K. Das, "A secure and effective biometric-based user authentication scheme for wireless sensor networks using smart card and fuzzy extractor," International Journal of Communication Systems, vol. 30, no. 1, pp. 1-25, 2017.

[6] J. Yan, Y. Meng, X. Yang, X. Luo, and X. Guan, "Privacypreserving localization for underwater sensor networks via deep reinforcement learning," IEEE Transactions on Information Forensics and Security, no. 99, p. 1, 2020.

[7] J. Duan, D. Gao, and Y. Dong, "An energy-aware trust derivation scheme with game theoretic approach in wireless sensor networks for IoT applications," IEEE Internet of Things Journal, vol. 1, no. 1, pp. 58-69, 2017.

[8] C. Zhan, Y. Zeng, and R. Zhang, "Energy-efficient data collection in UAV enabled wireless sensor network," IEEE Wireless Communication Letters, no. 99, p. 1, 2017.

[9] M. Jahanbakht, W. Xiang, L. Hanzo, and M. R. Azghadi, "Internet of Underwater Things and big marine data analytics - a comprehensive survey," IEEE Communications Surveys \& Tutorials, vol. 23, no. 2, pp. 904-956, 2021.

[10] J. Jiang, G. Han, and F. Wang, "An efficient distributed trust model for wireless sensor networks," IEEE Transactions on Parallel and Distributed Systems, vol. 26, no. 5, pp. 1228-1237, 2016.

[11] Y. Ma, "Research on the arrangement and visual design of aerobics under the new situation," International Core Journal of Engineering, vol. 5, no. 9, pp. 170-173, 2019.

[12] C. Benzaid, K. Lounis, A. Al-Nemrat, N. Badache, and M. Alazab, "Fast authentication in wireless sensor networks," Future Generation Computer Systems, vol. 55, no. FEB, pp. 362-375, 2016.

[13] A. Ts, D. Dmb, A. Xs, and B. Mfc, "Multi-sensor state estimation over lossy channels using coded measurements," Automatica, vol. 111, Article ID 108561, 2020.

[14] T. Takeshi, "Quantification of otolith functions and gravitational recognition using 3-dimensional eye movement analysis and a description of Listing's plane," Equilibrium Research, vol. 78, no. 3, pp. 197-202, 2019.

[15] J. . Hou, "Research on the feature description and behavior recognition of sports human body based on vision," Agro Food Industry Hi-Tech, vol. 28, no. 1, pp. 2636-2640, 2017.

[16] Y. Bai and Y. Chen, "Human motion analysis and action scoring technology for sports training based on computer vision features," Journal of Intelligent and Fuzzy Systems, vol. 34, pp. 1-9, 2021.

[17] Y. Chen, "Sports sequence images based on convolutional neural network," Mathematical Problems in Engineering, vol. 2021, no. 4, 14 pages, Article ID 3326847, 2021.

[18] S. Knig and S. Greve, "On the problems of honorary work in German sports clubs - a qualitative dominat-ed crossover mixed methods study," International Journal of Multiple Research Approaches, vol. 12, no. 3, pp. 335-353, 2021.

[19] H. Zhang, X. Hong, and J. Cheng, "Secure resource allocation for OFDMA two-way relay wireless sensor networks without and with cooperative jamming," IEEE Transactions on Industrial Informatics, vol. 12, no. 5, pp. 1714-1725, 2017.

[20] M. Dong, K. Ota, and A. Liu, "RMER: reliable and energyefficient data collection for large-scale wireless sensor networks," IEEE Internet of Things Journal, vol. 3, no. 4, pp. 511-519, 2017.
[21] Y. Deng, L. Wang, and M. Elkashlan, "Physical layer security in three-tier wireless sensor networks: a stochastic geometry approach," IEEE Transactions on Information Forensics and Security, vol. 11, no. 6, pp. 1128-1138, 2017.

[22] Z. P. Cai, Z. B. He, X. Guan, and Y. S. Li, "Collective datasanitization for preventing sensitive information inference attacks in social networks," IEEE Transactions on Dependable and Secure Computing, vol. 15, no. 4, pp. 577-590, 2018.

[23] X. Zheng and Z. Cai, "Privacy-preserved data sharing towards multiple parties in industrial iots," IEEE Journal on Selected Areas in Communications, vol. 38, no. 5, pp. 968-979, 2020.

[24] Y. Hu, M. Dong, and K. Ota, "Mobile target detection in wireless sensor networks with adjustable sensing frequency," IEEE Systems Journal, vol. 10, no. 3, pp. 1160-1171, 2017.

[25] F. Dobslaw, T. Zhang, and M. Gidlund, "End-to-End reliability-aware scheduling for wireless sensor networks," IEEE Transactions on Industrial Informatics, vol. 12, no. 2, pp. 758-767, 2016. 